\title{
A quasiparticle version of the Ginocchio S,D fermion pair algebra
}

\author{
K.T. Hecht \\ Physics Department, University of Michigan, Ann Arbor, MI 48109, USA
}

Received 30 November 1992

\begin{abstract}
The automorphisms of $S O(8)$ which exchange vector and spinor representations do not lead to new physically meaningful subgroup chains for the nuclear S,D fermion pair algebra; but a simpler transformation leads to a physically meaningful quasiparticle version of this algebra.
\end{abstract}

\section{Introduction}

Recently the automorphisms of the group $\mathrm{SO}(8)$ have been used to advantage in several branches of physics. The very special nature of $\mathrm{SO}(8)$ arises from the unique three-fold symmetry of its Dynkin diagram. This has been highlighted by Georgi in a chapter entitled "Automorphisms: Fun with $\mathrm{SO}(8)$ " in his book on Lie algebras ${ }^{1}$ ) and leads to the embedding of three distinct $S O(7)$ subgroups within $\mathrm{SO}(8)$. These have been used in particle physics in superstring and supergravity theories ${ }^{2}$ ) and in the study of octonionic superstring solitons ${ }^{3}$ ). Very recently they have been used to great advantage by Judd and Lister ${ }^{4-6}$ ) to explain many unexpected simple proportionalities and unexplained zeros in atomic $\mathrm{f}$-shell matrix elements. These successes have led to the question: Are there three distinct physically relevant $\mathrm{SO}(7)$ subgroups in the most important nuclear physics application of $\mathrm{SO}(8)$, the Ginocchio S,D fermion pair algebra ${ }^{7}$ ) and its outgrowth, the fermion dynamic symmetry model $\left.{ }^{8}\right)$ ?

The automorphisms of $\mathrm{SO}(8)$ interchange the three fundamental 8-dimensional irreducible representations of this group, the 8-vector defining representation (1000), and the two real spinor representations $\left(\frac{111 \frac{1}{22} 2}{2}\right)$ and $\left(\frac{11}{22} \frac{1}{2}-\frac{1}{2}\right)$, and lead to the transformation of the general representation $\left(\Omega_{1} \Omega_{2} \Omega_{3} \Omega_{4}\right)$ into $\left(\frac{1}{2}\left(\Omega_{1}+\Omega_{2}+\Omega_{3}-\Omega_{4}\right), \frac{1}{2}\left(\Omega_{1}+\right.\right.$ $\left.\Omega_{2}-\Omega_{3}+\Omega_{4}\right), \frac{1}{2}\left(\Omega_{1}-\Omega_{2}+\Omega_{3}+\Omega_{4}\right)$, $\left.\pm \frac{1}{2}\left(-\Omega_{1}+\Omega_{2}+\Omega_{3}+\Omega_{4}\right)\right)$. These three $\operatorname{SO}(8)$

Correspondence to: Dr. K.T. Hecht, Department of Physics, University of Michigen, 500 East University, MI 48109-1120 Ann Arbor, USA.

* It is a pleasure to dedicate this short contribution to Hans Weidenmüller on the occasion of his 60 th birthday. 
irreducible representations have the same dimension and the same values for the quadratic Casimir invariants, but differ markedly in the embedding of the three types of $\mathrm{SO}(7)$ irreducible representations ${ }^{9}$ ), which are given by the usual "betweenness" conditions for the quantum numbers. E.g., the generalized seniority-zero irrep. (3000) of the Ginocchio pair algebra leads to the D pair SO(7) irreps. $(\omega 00)$ with $\omega=3,2,1,0$. The associated $\operatorname{SO}(8)$ irreps. $\left(\frac{33}{22} \frac{3}{2} \pm \frac{3}{2}\right)$ on the other hand would lead to a single $\mathrm{SO}(7)$ irrep. $\left(\frac{33}{22}\right)$. Is this a physically relevant irrep. for the Ginocchio $\mathrm{S}, \mathrm{D}$ pair algebra? The mappings of the $\mathrm{SO}(8)$ generators into their counterparts under the automorphisms is straightforward ${ }^{1}$ ). To be physically relevant, however, for the nuclear physics applications these mappings would have to preserve the subgroup characteristics of the angular momentum $\mathrm{SO}(3)$ generators. In the atomic applications ${ }^{4-6}$ ) the 28 generators of $\mathrm{SO}(8)$ are constructed from spherical tensors of rank $r=1$ and 5 and two different tensors of rank 3 . The three distinct $\operatorname{SO}(7)$ subgroups are generated by the 21 operators which include the $r=1$ and 5 spherical tensors and three distinct linear combinations of the two independent $r=3$ spherical tensors. Since the $\mathrm{SO}(8)$ automorphism does not mix spherical tensors of different rank, the angular momentum subgroup generators remain intact. The nuclear S,D fermion pair algebra, on the other hand, is generated by $28 \mathrm{SO}(8)$ operators which decompose into single spherical tensors of ranks $r=1$ and 3 and three distinct spherical tensors of ranks $r=0$ and 2 each. To keep the angular momentum subgroup generators intact, the $S O(7)$ subgroups can only be generated by 21 operators which include the $r=1$ and 3 spherical tensors and linear combinations of the $r=2$ tensors and the $r=0$ tensors. The familiar D pair $\mathrm{SO}(7)$ subalgebra ${ }^{7,10}$ ) of the S,D pair $S O(8)$ algebra is generated by the $5 \mathrm{D}$ pair creation operators, their companion $\mathrm{D}$ pair annihilation operators, the nucleon number operator which is an $r=0$ operator, and the $r=1$ and 3 spherical tensors. It is the purpose of this note to show that there are other realizations of the $\mathrm{SO}(8) \supset \mathrm{SO}(7)$ algebra corresponding to different linear combinations of the $r=2$ and 0 tensors. These can be interpreted as quasiparticle realizations of the $S O(8)$ algebra. Their $S O(7)$ subalgebras correspond to quasi-particle realizations of the D pair algebra and they are not generated via the transformations of the $\mathrm{SO}(8)$ automorphisms which exchange vector and spinor representations. In the nuclear application these automorphisms would mix spherical tensors of different rank. Despite the failure of the $S O(8)$ automorphism to lead to new physics in the case of the nuclear S,D pair algebra, the new quasi-particle realizations of the Ginocchio $\mathrm{SO}(8)$ algebra may be of special interest in their own right. This $S O(8)$ algebra applies to fermion pairs created from identical nucleons in the normal parity orbits of the shell model; e.g., the $s_{1 / 2}, d_{3 / 2}, d_{5 / 2}$ and $g_{7 / 2}$ orbits for $50 \leqslant N \leqslant 82$. Since the nucleon occupation probabilities for these orbits must compete with the wrong-parity $h_{11 / 2}$ intruder, a normal-parity pair model built from quasi-particles rather than particles may lead to the physically more relevant symmetry. 


\section{The $S, D$ pair algebra revisited}

In the Ginocchio S,D fermion pair model the single-nucleon creation operators with definite total angular momentum quantum numbers $j, m$ are expressed in terms of single particle pseudo orbital $(k)$ and pseudo-spin $(i)$ angular momenta, such that $\boldsymbol{k}+\boldsymbol{i}=\boldsymbol{j}$,

$$
a_{j m}^{\dagger}=\sum_{m_{k}, m_{i}}\left\langle k m_{k} i m_{i} \mid j m\right\rangle a_{k m_{k}, m_{i}}^{\dagger} .
$$

With $i=\frac{3}{2}$ (the $\mathrm{SO}(8)$ algebra) the single particle quantum numbers $j$ can take on the values,

$$
\begin{array}{ll}
\text { with } k=1: & j=\frac{1}{2}, \frac{3}{2}, \frac{5}{2} \quad(\Omega=6), \\
\text { with } k=2: & j=\frac{1}{2}, \frac{3}{2}, \frac{5}{2}, \frac{7}{2} \quad(\Omega=10),
\end{array}
$$

e.g. the normal-parity orbits of the nuclear p,f shells $(28 \leqslant N \leqslant 50)$ or $s, d, g$ shells $(50 \leqslant N \leqslant 82)$, with $\Omega=\Sigma_{j}\left(j+\frac{1}{2}\right)=2(2 k+1)$. The $\mathrm{SO}(8)$ algebra is generated by the fermion pair operators coupled to angular momentum zero in the pseudo orbital $k$-space

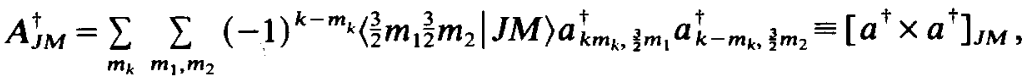

$$
\begin{aligned}
& A_{J M}=\left(A_{J M}^{\dagger}\right)^{\dagger} \\
& C_{\mu}^{r}=\left\{\sum_{m_{k}} \sum_{m_{1}, m_{2}}\left\langle\frac{3}{2} m_{12}^{3}-m_{2} \mid r \mu\right\rangle a_{k m_{k}, \frac{3}{2} m_{1}}^{\dagger} a_{k m_{k} \frac{3}{2} m_{2}}(-1)^{\frac{3}{2}-m_{2}}\right\}-\frac{1}{2} \Omega \delta_{r o} \\
& \equiv\left[a^{\dagger} \times \tilde{a}\right]_{\mu}^{r}-\frac{1}{2} \Omega \delta_{r o},
\end{aligned}
$$

where the pair creation and annihilation operators, $A_{J M}^{\dagger}, A_{J M}$ are restricted to $J=0$, 2 only (S,D pairs), while the one-body operators $C_{\mu}^{r}$ range through $r=0,1,2,3$ leading to the spherical tensor classification of the $\mathrm{SO}(8)$ generators enumerated in the introduction. Note also that $C_{0}^{0}=\frac{1}{2} N_{\mathrm{op}}-\frac{1}{2} \Omega$, where $N_{\mathrm{op}}$ is the nucleon number operator. The 28 group generators $A_{J M}^{\dagger}, A_{I M}, C_{\mu}^{r}$ are put into $1: 1$ correspondence with standard $\mathrm{SO}(8)$ generators $J_{a b}=-i\left(x_{a} \partial_{b}-x_{b} \partial_{a}\right)$ with $a, b=1, \ldots, 8$ through table 1 of ref. $\left.{ }^{10}\right)$. This $\mathrm{SO}(8)$ group has two physically relevant $\mathrm{SO}(n)$ subgroup chains,

$$
\left.\begin{array}{c}
\mathrm{SO}(8) \supset \mathrm{SO}(7) \\
\mathrm{SO}(8)>\mathrm{SO}(6) \times \mathrm{SO}(2)
\end{array}\right\} \supset \mathrm{SO}(5) \times \mathrm{SO}(2) \supset \mathrm{SO}(3) \times \mathrm{SO}(2),
$$

The $S O(7)$ subgroup is generated by the $D$ pair creation and annihilation operators $A_{2 M}^{\dagger}, A_{2 M}$ and the one body-operators, $C_{\mu}^{r}$, with $r=0,1$ and 3 where these can be put into $1: 1$ correspondence ${ }^{10}$ ) with $J_{a b}$, with $a, b=1, \ldots, 7$ while the subgroup $\mathrm{SO}(6)$ is generated by the $C_{\mu}^{r}$ with $r=1,2,3$ corresponding to $J_{a b}$, with $a, b=1, \ldots, 5$ and 8 . Note that these commute with $C_{0}^{0}$ corresponding to the $\mathrm{SO}(2)$ generator $J_{67}$, leading to the $\mathrm{SO}(6) \times \mathrm{SO}(2)$ subgroup which can be used in the full classification of the $\operatorname{SO}(8)$ irreducible representations $\left(\Omega_{1} \Omega_{2} \Omega_{3} \Omega_{4}\right)=\left(\frac{1}{2} \Omega-\frac{1}{2} u, \rho_{1} \rho_{2} \rho_{3}\right)$. Here $\Omega_{1}$ 
is given by the highest possible weight of $C_{0}^{0}$ corresponding to maximum nucleon number $2 \Omega-u$, where $u$, the generalized S,D pair seniority number, is the number of nucleons entirely free of the favored $S$ and $D$ pairs of eq. (2). The remaining $\mathrm{SO}(8)$ quantum numbers $\rho_{1}, \rho_{2}, \rho_{3}$ give the $\mathrm{SO}(6)$ symmetry of the $u$ nucleons free of favored $\mathrm{S}$ and $\mathrm{D}$ pairs. The full set of states of the irrep., $\left(\frac{1}{2} \Omega-\frac{1}{2} u, \rho_{1} \rho_{2} \rho_{3}\right)$, with nucleon numbers $n=u, u+2, \ldots, 2 \Omega-u-2,2 \Omega-u$ can then be generated by coupling $p=\frac{1}{2}(n-u)$ favored nucleon S,D pairs with $\mathrm{SO}(6)$ labels $(p 00),(p-$ $2,00), \ldots,(100)$ or $(000)$, resulting from $p$ symmetrically coupled $\mathrm{SO}(6)(100)$ pairs, with the $S O(6)$ representations $\left(\rho_{1} \rho_{2} \rho_{3}\right)$ of the $u$ nucleons free of favored S,D pairs to resultant $\mathrm{SO}(6)$ representations $\left(\sigma_{1} \sigma_{2} \sigma_{3}\right)$. Table 1 shows a very simple example, the $\mathrm{SO}(8)$ irrep. (3000) corresponding to the states of generalized seniority $u=0$ in the $\Omega=6$ shell. Finally, the subgroup $\mathrm{SO}(5)$ common to both group chains of eq. (5) is generated by the $C_{\mu}^{r}$ with $r=1$ and 3 corresponding to $J_{a b}$ with $a, b=1, \ldots, 5$, whereas the final $\mathrm{SO}(3)$ subgroup is generated by the $C_{\mu}^{r}$ with $r=1$. Its irreps. give the I-spin and in this subspace of states with a zero pseudo-orbital angular momentum, the total angular momentum, $J$, of the states. This is shown as a right subscript on the $\mathrm{SO}(5)$ irreps $\left(\tau_{1} \tau_{2}\right)$ of table 1 . The $\mathrm{SO}(7)$ irreps. $\left(\omega_{1} \omega_{2} \omega_{3}\right)=\left(\frac{1}{2} \Omega-\frac{1}{2} w, \theta_{1} \theta_{2}\right)$ embedded within $\left(\Omega_{1} \Omega_{2} \Omega_{3} \Omega_{4}\right)$ are given by the "betweenness" conditions: $\Omega_{1} \geqslant \omega_{1} \geqslant$ $\Omega_{2}, \Omega_{2} \geqslant \omega_{2} \geqslant \Omega_{3}, \Omega_{3} \geqslant \omega_{3} \geqslant\left|\Omega_{4}\right|$. Now, $w$ is the $\mathrm{D}$ pair seniority number which counts the number of nucleons entirely free of favored D pairs (note that $w \geqslant u$ ). In the example of table 1 the 2-particle state generated by the action of $A_{00}^{+}$is an SO(7) state, (200), with $w=2$; while the 4-particle state generated by the action of two pair-creation operators $A_{00}^{\dagger}$ is in the $\mathrm{SO}(7)$ irrep. (100) with $w=4$, etc. States with larger particle numbers in these $S O(7)$ irreps. are then generated by the action of $p=\frac{1}{2}(n-w)$ D pair-creation operators with $S O(5)$ symmetries $(p 0),(p-2,0), \ldots$, as shown specifically in table 2 . The $\operatorname{SO}(5)$ labels $\left(\tau_{1}, \tau_{2}\right)$ are shown as right superscripts whereas the angular momentum labels are given by right subscripts in this table. States with $n>6$ (more than half-full shell) are given by the hole equivalents, involving pair annihilation operators acting on the full shell state.

TABLE 1

States of the $\mathrm{SO}(8)$ irrep. (3000) in the $\mathrm{SO}(8) \supset \mathrm{SO}(6) \times \mathrm{SO}(2) \supset \mathrm{SO}(5) \times \mathrm{SO}(2) \supset \mathrm{SO}(3) \times \mathrm{SO}(2)$ chain

\begin{tabular}{ccl}
\hline $\begin{array}{c}\mathrm{SO}(2) \\
n\end{array}$ & \multicolumn{1}{c}{$\mathrm{SO}(6)$} \\
$\left(\sigma_{1} \sigma_{2} \sigma_{3}\right)$ & \multicolumn{1}{c}{\begin{tabular}{c}
\multicolumn{1}{c}{$\mathrm{SO}(5) \supset \mathrm{SO}(3)$} \\
$\left(\tau_{1} \tau_{2}\right)_{J}$
\end{tabular}} \\
\hline 12 & $(000)$ & $(00)_{J=0}$ \\
10 & $(100)$ & $(10)_{J=2}(00)_{J=0}$ \\
8 & $(200)(000)$ & $(20)_{J=2,4}(10)_{J-2}(00)_{J=0}^{2}$ \\
6 & $(300)(100)$ & $(30)_{J=0,3,4,6}(20)_{J=2,4}(10)_{J=2}^{2}(00)_{J=0}^{2}$ \\
4 & $(200)(000)$ & $(20)_{J=2,4}(10)_{J=2}(00)_{J=0}^{2}$ \\
2 & $(100)$ & $(10)_{J-2}(00)_{J-0}$ \\
0 & $(000)$ & $(00)_{J=0}$ \\
\hline
\end{tabular}


TABLE 2

State vectors of $\mathrm{SO}(7)$ irreps. $(\omega 00)$ embedded within the $\mathrm{SO}(8)$ irrep. (3000)

\begin{tabular}{|c|c|c|c|}
\hline$(300)$ & $(200)$ & $(100)$ & $(000)$ \\
\hline $\begin{array}{c}\mid \text { Full }\rangle \\
\left.A_{2 M}^{(10)} \mid \text { Full }\right\rangle \\
\left.\left[A_{2} \times A_{2}\right]_{j=0,2,4}^{(00)} \mid \text { Full }\right\rangle \\
{\left[A_{2}^{+} \times A_{2}^{+} \times A_{2}^{+}\right]_{j=2,(0,3,4,6}^{(10),(30)}|0\rangle} \\
{\left[A_{2}^{+} \times A_{2}^{\dagger}\right]_{j=0,2,4}^{(00)}|0\rangle} \\
A_{2 M}^{+(10)}|0\rangle \\
|0\rangle\end{array}$ & $\begin{array}{c}\left.A_{00}^{(00)} \mid \text { Full }\right\rangle \\
\left.\left[A_{2} \times A_{0}\right]_{2 M}^{(10)} \mid \text { Full }\right\rangle \\
\left.\left[A_{2}^{\dagger} \times A_{2}^{\dagger} \times A_{0}^{\dagger}\right]_{j=0,2,4}^{(0)}(0), 0\right\rangle \\
{\left[A_{2}^{\dagger} \times A_{0}^{\dagger}\right]_{2 M,}^{(10)}|0\rangle} \\
A_{00}^{+(00)}|0\rangle\end{array}$ & $\begin{array}{c}\left.\left[A_{0} \times A_{0}\right]_{00}^{(00)} \mid \text { Full }\right\rangle \\
{\left[A_{2}^{+} \times A_{0}^{\dagger} \times A_{0}^{+}\right]_{2 M}^{(10)}|0\rangle} \\
{\left[A_{0}^{\dagger} \times A_{0}^{\dagger}\right]_{00}^{(00)}|0\rangle}\end{array}$ & {$\left[A_{0}^{\dagger} \times A_{0}^{\dagger} \times A_{0}^{\dagger}\right]_{00}^{(00)}|0\rangle$} \\
\hline
\end{tabular}

The question to be examined is now the following: Are there different physically relevant subgroups $\mathrm{SO}(7)$ or $\mathrm{SO}(6)$ in the S,D fermion-pair algebra, i.e. are there different subgroups generated by linear combinations of the $\mathrm{SO}(8)$ generators which preserve the spherical tensor character of these operators and contain the $\mathrm{SO}(3)$ subgroup generators $C_{\mu}^{r}$ with $r=1$. Clearly this will have to be limited to linear combinations of the $r=2$ and 0 tensors. The following is a solution. The S,D pair-creation operators can be replaced by the two combinations

$$
U^{2} A_{J M}^{\dagger}-V^{2} A_{J-M}(-1)^{J-M} \mp 2 U V C_{M}^{J}
$$

with $U^{2}+V^{2}=1$.

If the D pair-creation operators of the standard $\mathrm{SO}(7)$ subgroup of $\mathrm{SO}(8)$ are replaced by the $J=2$ operators of eq. (6) we are led to the two new $\operatorname{SO}(7)$ subgroups, $\mathrm{SO}(7)^{\prime}$ and $\mathrm{SO}(7)^{\prime \prime}$. However, these are not related to the automorphisms of $\mathrm{SO}(8)$ which exchange vector and spinor irreps. Instead they are merely quasiparticle realizations of the standard $\mathrm{SO}(8) \supset \mathrm{SO}(7)$ group. For simplicity only the case for the upper sign of eq. (6) will be discussed. This arises from the quasiparticle transformation

$$
b_{k m_{k}, \frac{3}{2} m_{i}}^{\dagger}=U a_{k m_{k}, \frac{3}{2} m_{i}}^{\dagger}-V a_{k-m_{k}, \frac{3}{2}-m_{i}}(-1)^{k+m_{k}+\frac{1}{2}+m_{i}} .
$$

Using the shorthand notation introduced in eqs. (2) and (4) the operators of eq. (6) are simply $\left[b^{\dagger} \times b^{\dagger}\right]_{M}$, again with $J=2,0$. Together with the conjugate $[\tilde{b} \times \tilde{b}]_{J M}$ and the operators $\left(\left[b^{\dagger} \times \tilde{b}\right]_{\mu}^{r}-\frac{1}{2} \Omega \delta_{r 0}\right)$ they generate a new copy of $\operatorname{SO}(8)$. The $\left[b^{\dagger} \times \tilde{b}\right]_{\mu}^{r}$ operators are simply:

For $r=$ odd $(r=1,3)$ :

$$
\left[b^{\dagger} \times \tilde{b}\right]_{\mu}^{r}=\left[a^{+} \times \tilde{a}\right]_{\mu}^{r} .
$$

For $r=$ even $(r=0,2)$ :

$$
\left[b^{\dagger} \times \tilde{b}\right]_{\mu}^{r}-\frac{1}{2} \Omega \delta_{r 0}=U V\left(A_{r \mu}^{\dagger}+(-1)^{r-\mu} A_{r-\mu}\right)+\left(U^{2}-V^{2}\right) C_{\mu}^{r} .
$$


The SO $(8)$ irreps. generated by the 28 operators $\left[b^{\dagger} \times b^{\dagger}\right]_{M},[\tilde{b} \times \tilde{b}]_{M},\left(\left[b^{\dagger} \times \tilde{b}\right]_{\mu}^{r-}\right.$ $\left.\frac{1}{2} \Omega \delta_{r 0}\right)$ can still be labelled by $\left(\frac{1}{2} \Omega-u^{\prime}, \rho_{1}^{\prime} \rho_{2}^{\prime} \rho_{3}^{\prime}\right)$, where $u^{\prime}$ is now the number of quasiparticles entirely free of favored $S$ and $D$ pair quantum numbers. With $u^{\prime}=0$ and $\Omega=6$ the complete set of states can still be given by table 2 if the $a^{\dagger}$ are replaced by $b^{\dagger}$ and the true vacuum, $|0\rangle$, is replaced by the quasiparticle vacuum for the $U$ 's and $V$ 's of eq. (7). The full $\mathrm{SO}(8)$ irrep. (3000) still breaks apart into four $\mathrm{SO}(7)^{\prime}$ irreps $(300),(200),(100)$ and $(000)$. The $\mathrm{SO}(7)^{\prime}$ generators do not mix states in the quasiparticle analogs of different columns of table 2 . Note that this is very different from the groups $\mathrm{SO}(7)^{\prime}$ and $\mathrm{SO}(7)^{\prime \prime}$ generated by the automorphisms of $\mathrm{SO}(8)$ which exchange vector and spinor representations. For both of these the generalized $u=0$ state for $\Omega=6$ would be spanned by a single 112 -dimensional irrep. $\left(\frac{33}{2} \frac{3}{2}\right)$ of $\mathrm{SO}(7)^{\prime}$ or $\mathrm{SO}(7)^{\prime \prime}$. The action of $\mathrm{SO}(7)^{\prime}$ generators would thus have to mix all states of table 2 .

Finally, it may be instructive to exhibit explicitly the particle character of the $S O(7)^{\prime}$ states. For this purpose we choose the four states with $J=4$ in the irrep. (3000). The 3 states of the $\mathrm{SO}(7)^{\prime}$ irrep (300) are given by:

$$
\begin{aligned}
|\tilde{n}=8\rangle= & -V^{2} \mathcal{N}\left[A_{2}^{\dagger} \times A_{2}^{\dagger}\right]_{J=4, M}|0\rangle-\sqrt{2} U V \mathcal{N}^{\prime}\left[A_{2}^{\dagger} \times A_{2}^{\dagger}\right]_{4 M} \times A_{00}^{\dagger}|0\rangle \\
& \left.+U^{2} \mathcal{N}\left[A_{2} \times A_{2}\right]_{4,-M} \mid \text { Full }\right\rangle, \\
|\tilde{n}=6\rangle= & \mathcal{N}^{\prime \prime}\left[A_{2}^{\dagger} \times A_{2}^{\dagger} \times A_{2}^{\dagger}\right]_{J=4, M}|0\rangle, \\
|\tilde{n}=4\rangle= & U^{2} \mathcal{N}\left[A_{2}^{\dagger} \times A_{2}^{\dagger}\right]_{J=4, M}|0\rangle-\sqrt{2} U V \mathcal{N}^{\prime}\left[A_{2}^{\dagger} \times A_{2}^{\dagger}\right]_{4 M} \times A_{00}^{\dagger}|0\rangle \\
& \left.-V^{2} \mathcal{N}\left[A_{2} \times A_{2}\right]_{4,-M} \mid \text { Full }\right\rangle .
\end{aligned}
$$

For the $S O(7)^{\prime}$ irrep. (200):

$$
\begin{aligned}
|\tilde{n}=6\rangle= & \sqrt{2} U V \mathcal{V N}\left[A_{2}^{\dagger} \times A_{2}^{\dagger}\right]_{J=4, M}|0\rangle+\left(U^{2}-V^{2}\right) \mathcal{N}^{\prime}\left[A_{2}^{\dagger} \times A_{2}^{\dagger}\right]_{4 M} \times A_{00}^{\dagger}|0\rangle \\
& \left.+\sqrt{2} U V \mathcal{N}\left[A_{2} \times A_{2}\right]_{4,-M} \mid \text { Full }\right\rangle,
\end{aligned}
$$

where $\tilde{n}$ denotes the eigenvalue of $\left[b^{\dagger} \times \tilde{b}\right]_{0}^{0}$ and the $\mathcal{N}, \mathcal{N}^{\prime}, \mathcal{N}^{\prime \prime}$ are trivial normalization constants.

\section{Discussion}

The transformations associated with the automorphisms of $\mathrm{SO}(8)$ which transform the 8-dimensional vector representation $(1000)$ into the spinor representations $\left(\frac{111}{22} \frac{1}{2}\right)$ or $\left(\frac{111}{22}-\frac{1}{2}\right)$ unfortunately do not lead to physically relevant distinct subgroups $\mathrm{SO}(7)$, $\mathrm{SO}(7)^{\prime}, \mathrm{SO}(7)^{\prime \prime}$ for the Ginocchio S,D fermion pair algebra. These transformations place pieces of the angular momentum algebra outside the subgroups $\operatorname{SO}(7)^{\prime}$ or $\mathrm{SO}(7)^{\prime \prime}$ so that states of irreps. of these groups no longer carry good angular momentum, a basic requirement for a physically relevant subgroup. Despite the failure of these $\operatorname{SO}(8)$ automorphisms to lead to new physics in the case of the nuclear S,D pair algebra, there are now simpler transformations which mix the 
spherical tensors of rank 2 and 0 of this algebra to lead to new subgroups $\mathrm{SO}(7)^{\prime}$ and $\mathrm{SO}(7)^{\prime \prime}$ which are, however, simple copies of the original subgroup chains, with similar irreducible representation embeddings. The mixing coefficients $U$ and $V$ can be given a simple quasiparticle interpretation. The irreducible representation labels for the quasiparticle realization are to be interpreted as generalized quasiparticle seniority quantum numbers in place of the usual real particle seniority numbers. The SO(8) S,D pair algebra applies to fermion pairs created from identical nucleons in the normal parity orbits of the shell model; with $k=2, i=\frac{3}{2}$, e.g., the $\mathrm{s}_{1 / 2}, \mathrm{~d}_{3 / 2}, \mathrm{~d}_{5 / 2}$ and $g_{7 / 2}$ orbits for $50 \leqslant N \leqslant 82$. Since the nucleon occupation probabilities for these orbits must compete with the abnormal parity $h_{11 / 2}$ intruder orbit, a normal parity pair model built from quasiparticles rather than particles may lead to the physically more meaningful symmetry.

The Ginocchio S,D pair model was originally introduced as a toy model to study the fermionic foundation of the interacting boson model of Arima and Iachello. More recently it has been proposed as a fermion dynamical symmetry model meriting serious consideration for nuclear spectra throughout the periodic chart ${ }^{8}$ ). Since the Ginocchio S,D pair algebra is based on fermion pairs built from normal parity shell model orbits only, it fails to properly take into account the contributions of the abnormal parity high- $j$ intruder orbits. By making a transformation to quasiparticle $\mathrm{S}$ and $\mathrm{D}$ pairs with $U^{2}$ and $V^{2}$ which give the occupation probabilities of the essentially degenerate normal-parity orbits of a major shell, the quasiparticle version of this fermionic pair model permits a distribution of occupation probabilities among the abnormal parity orbits as well as the orbits above and below the valence shells. The quasiparticle version of the $\mathrm{SO}(8)$ algebra may thus alleviate one of the shortcomings of the fermion dynamic symmetry model. Since the new version of the algebra does not change the angular momentum characteristics of the favored D pair it will, however, not cure one of the serious problems of this model. Since the favored D pair of the $S O(8)$ algebra has a $j_{1}-j_{2}$ structure quite different from that predicted for realistic low seniority $2^{+}$states in real nuclei ${ }^{11,12}$ ), the successes of the fermion dynamic symmetry model ${ }^{8}$ ) may involve a mapping from real space onto a model space which is quite as complicated as the mapping from fermion space onto a boson space which is the basis for the interacting boson model of Arima and Iachello.

\section{References}

1) H. Georgi, Lie algebras in particle physics (Addison-Wesley, Reading, MA, 1982) ch. XXV

2) R. Dündarer, F. Gursey and C.H. Tze, Nucl. Phys. B266 (1986) 440

3) J.A. Harvey and A. Strominger, Phys. Rev. Lett. 66 (1991) 549

4) B.R. Judd and G.M.S. Lister, Phys. Rev. Lett. 67 (1991) 1720

5) B.R. Judd and G.M.S. Lister, J. de Phys. II 2 (1992) 853

6) B.R. Judd and G.M.S. Lister, in Group theory and special symmetries in nuclear physics, eds. J. Jänecke and J.P. Draayer (World Scientific, Singapore, 1992) pp. 225, 258

7) J.N. Ginocchio, Ann. of Phys. 126 (1980) 234 
8) J.Q. Chen, D.H. Feng and C.L. Wu, Phys. Rev. C34 (1986) 2269;

C.L. Wu, D.H. Feng, X.G. Chen, J.Q. Chen and M.W. Guidry, Phys. Rev. C36 (1987) 1157

9) B.G. Wybourne, J. of Phys. B 25 (1992) 1683

10) K.T. Hecht, Nucl. Phys. A475 (1987) 276

11) P. Halse, Phys. Rev. C36 (1987) 372

12) Sudha Swaminathan, University of Michigan dissertation (1993) unpublished 